$\begin{array}{cl}\begin{array}{c}\text { Revue } \\ \text { de /histoire }\end{array} & \text { Revue de l'histoire des religions } \\ \text { des religions } & \begin{array}{l}3 \mid 2015 \\ \text { Varia }\end{array}\end{array}$

\title{
Une autre forme de procès. La vérité et le droit dans l'exégèse du Nouveau Testament
}

Other Trials to come. Truth and the Law in New Testament Exegesis

\section{Philippe Büttgen}

\section{(2) OpenEdition \\ Journals}

Édition électronique

URL : http://journals.openedition.org/rhr/8407

DOI : $10.4000 /$ rhr.8407

ISSN : 2105-2573

Éditeur

Armand Colin

Édition imprimée

Date de publication : 1 septembre 2015

Pagination : $325-338$

ISBN : 978-2-200-92993-0

ISSN : 0035-1423

Référence électronique

Philippe Büttgen, «Une autre forme de procès. La vérité et le droit dans l'exégèse du Nouveau

Testament », Revue de l'histoire des religions [En ligne], 3 | 2015, mis en ligne le 01 septembre 2018

consulté le 06 mai 2019. URL : http://journals.openedition.org/rhr/8407 ; DOI : 10.4000/rhr.8407 


\section{PHILIPPE BÜTTGEN}

Université Paris 1 - Panthéon-Sorbonne

Centre de philosophie contemporaine de la Sorbonne

\section{Une autre forme de procès La vérité et le droit dans l'exégèse du Nouveau Testament}

Qu'y a-t-il derrière la vérité (alêtheia) dans le Nouveau Testament? Aucune "conception" particulière, pas même dans les textes johanniques, aucune définition dissidente de la vérité, simplement la vérité la plus ordinaire: l'adéquation de la chose et de la pensée. Les textes bibliques, cependant, font voir ce qu'il y a derrière l'adéquation: le droit, mais aussi une critique complexe et impitoyable des catégories du droit, témoignage, déposition, tribunal. À partir d'une lecture de Jn 8 et 18, on discute les effets de presque un siècle d'interprétation bultmannienne, ainsi que les conséquences de l'étrange tournant ecclésiologique qu'a pris la philosophie depuis une vingtaine d'années.

\section{Other Trials to come. Truth and the Law in New Testament Exegesis}

What stands behind truth (alêtheia) in the New Testament? No particular 'conception' of truth, even not in Johannine texts, no extraordinary definition - simply truth, in its most ordinary meaning, as the adequation of thoughts to things. Biblical texts, however, give access to what stands behind this adequation: the law, as well as a complex and harsh criticism of the categories of the law - charges, testimony, tribunal. Starting from a reading of John 8 and 18, I discuss the consequences of almost one century of Bultmannian interpretation of the New Testament, as well as the strange ecclesiological turn that has taken place over the past twenty years in continental philosophy. 
Les recherches actuelles en exégèse mettent l'accent sur le caractère presque parfaitement ordinaire des conceptions de la vérité dans le Nouveau Testament ${ }^{1}$. Le constat est fade et presque décevant après un demi-siècle de prévalence heideggérienne. Après l'alêtheia, l' «authentique possibilité du Dasein» et le «dualisme de la décision», après la «conception johannique de la vérité», l'exhaustion de ses sources juives, grecques, gnostiques, en un mot: après Bultmann ${ }^{2}$, et après encore vingt ans de spéculation sur la « vérité selon le christianisme ${ }^{3}$, faudra-t-il en revenir, pour la Bible aussi, à nos définitions scolaires de la vérité, à la banale adéquation de la pensée et de la chose?

J'aimerais voir où mène ce sens retrouvé de l'ordinaire biblique: celui d'une religion qui revendiquerait la vérité comme tout le monde le fait, en affirmant simplement que ce qu'elle dit correspond à ce qui est. Les motivations de l'exégèse laissent souvent à désirer ${ }^{4}$. Il nous suffira de quelques indices pour commencer.

1. Voir Christof Landmesser, Wahrheit als Grundbegriff neutestamentlicher Wissenschaft, Tübingen, Mohr Siebeck (Wissenschaftliche Untersuchungen zum Neuen Testament, 113), 1998; Id., art. "Wahrheit/Wahrhaftigkeit», II. Neues Testament [in] Theologische Realenzyklopädie, t. 35, Berlin-New York, de Gruyter, 2003, p. 340-345, et l'important bilan de la recherche par Walter Schmithals, Johannesevangelium und Johannesbriefe. Forschungsgeschichte und Analyse, Berlin-New York, de Gruyter (Zeitschrift für die Neutestamentliche Wissenschaft, Beihefte, 64), 1992, part. p. 164-196.

2. Je renvoie sans développer à Rudolf Bultmann, «Untersuchungen zum Johannesevangelium», A. A $\lambda \hat{\eta} \theta \varepsilon ı \alpha$ (1928) [in] Id., Exegetica. Aufsätze zur Erforschung des Neuen Testaments, Tübingen, Mohr Siebeck, 1967, p. 124-

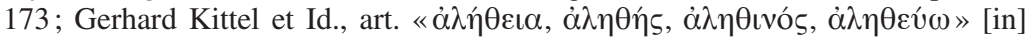
G. Kittel (dir.), Theologisches Wörterbuch zum Neuen Testament, t. 1, Stuttgart, Kohlhammer, 1933, p. 239-251; R. Bultmann, Das Evangelium des Johannes (1937-1941), Göttingen, Vandenhoeck \& Ruprecht (Kritisch-exegetischer Kommentar über das Neue Testament, 2), 1986'1 , p. 140-142, 243-244, 332-335, 468-469, 506-507; Id., Theologie des Neuen Testaments (1948-1953), Tübingen, Mohr Siebeck, $1984^{9}$, p. 71, 183-185, 368-373, 378-379.

3. Michel Henry, C'est moi la vérité. Pour une philosophie du christianisme, Paris, Le Seuil, 1996, p. 9-10 et passim. On préférera la méditation apaisée


Strasbourg, 36, 2014, p. 197-214.

4. La critique de Bultmann chez Landmesser est menée d'un point de vue intégralement métathéologique. Elle relève dans le concept «existentialontologique» de la vérité une «sous-détermination sémantique» censée faire obstacle au déploiement d'une véritable science néo-testamentaire (Ch. Land- 
Ce qui, sur un mode non strictement terminologique, désigne la vérité dans la Bible hébraïque (racine ' $m n$ ) résulte en plusieurs endroits d'un établissement des faits ${ }^{5}$, ou d'une correspondance entre un constat et des représentations ${ }^{6}$. Bultmann appelait cela bizarrement le sens «grec» de l'alêtheia: l' «état réel des choses », der "wirkliche" Tatbestand, alors qu'il semble bien plus que grec".

Il est intéressant d'observer la vérité-adéquation dans un tel environnement non spéculatif, «courant» si l'on y tient ${ }^{8}$, mais non moins pensant: l'adéquation sans sa définition, l'adéquation sans l'adaequatio (rei et intellectus), poursuivant des objectifs qui ne se soucient pas de philosophie. Les textes religieux ouvrent le chantier d'une archéologie de la vérité comme adéquation, au sens le plus strict d'une archéologie: non pas celui d'un retour à une quelconque origine - et pour cause, s'agissant de textes comme ceux du Tanakh, dont la rédaction s'est échelonnée sur des siècles pour se clore souvent fort tard, dans une chronologie très largement étrangère à l'histoire de la philosophie -, mais au sens d'une excavation qui charrie son lot de matériaux d'usage, parfois parfaitement banals.

messer, Wahrheit als Grundbegriff neutestamentlicher Wissenschaft, op. cit., p. 316, 327, cf. 104). Ce faisant, Landmesser rabat brutalement l'élucidation du concept biblique de vérité sur celle du concept de vérité nécessaire selon lui à l'édification d'une exégèse scientifique.

5. Gn 42, 15-20, épreuve imposée par Joseph à ses frères: «vos dires seront éprouvés: la vérité serait-elle avec vous?» Je cite d'après la Traduction Ecuménique de la Bible.

6. 1 R 10, 6, visite de la reine de Saba au roi Salomon: «C'était bien la vérité que j'avais entendu dire dans mon pays sur tes paroles et sur ta sagesse».

7. R. Bultmann, art. « $\dot{\alpha} \lambda \eta \dot{\theta} \theta \varepsilon \iota \alpha »$, loc. cit., p. 239-240, avec une rapide concession: ce que le Tanakh ignore serait seulement la «question de la vérité», la «question de l'étant véritable comme tel». Le point de rencontre entre l' 'emet hébraïque et l'alêtheia ne résiderait toutefois selon Bultmann que dans la qualification de la vérité comme norme d'action. Les versets cités dans les notes qui précèdent montrent cependant que l'intersection est plus vaste et qu'elle inclut une conception de la vérité comme adéquation. On peut voir aussi Diethelm Michel, "Ämät. Untersuchung über "Wahrheit" im Hebräischen», Archiv für Begriffsgeschichte 12 (1968), p. 30-57.

8. Martin Heidegger, Vom Wesen der Wahrheit (1930/1943), Frankfurt a. M., Klostermann, $1967^{5}$, p. 74 (= GA 9, p. 178), tr. fr. A. de Waehlens et W. Biemel [in] Questions I, Paris, Gallimard, 1968, p. 163. Heidegger varie les formules («Der geläufige Begriff der Wahrheit», mais aussi «gemeingültig», «gewohnt», «gangbar», ainsi que le lexique de l'«évidence» et du «sens commun», «Selbstverständlichkeit» et «gemeine[r] (Menschen)Verstand»), sans se préoccuper du sens précis de ce sens «courant». Une exception significative se trouve dans l'aperçu ibid., p. 76-77, tr. fr. p. 165-166, sur l'origine «théologique» de la doctrine de l'adaequatio. 
C'est, me semble-t-il, une des incitations les plus puissantes à lire la Bible en dehors de toute autre préoccupation: creuser le plus large écart possible entre la philosophie et la religion, à partir du concept le plus partagé de la vérité comme adéquation.

Retravailler l'adéquation à l'intérieur des définitions les plus ordinaires de la vérité: tel serait le projet. Que peut-on en attendre? On va le voir: le droit au cœur du vrai, comme ultima ratio de l'adéquation, mais aussi le contraire du droit. Commençons par les aspects techniques de la discussion.

\section{Témoignage}

Depuis Schlatter et von Soden, le débat exégétique se concentre autour de la question de savoir si l'usage d'alêtheia dans le corpus des écrits johanniques peut être ou non ramené aux autres usages bibliques et plus généralement à la signification ordinaire, "sémantiqueontologique», de la vérité ${ }^{9}$. Un interprète comme Landmesser argumente aujourd'hui ainsi: le concept johannique de la vérité comme logos fait chair en Jésus-Christ se distingue certes matériellement au sein des écrits bibliques (il statue différemment quant à ce qui est vrai), mais il dépend toujours formellement de la définition qui assimile ce qu'est le vrai à la correspondance entre une pensée, ou un énoncé, et ce qui est ${ }^{10}$. Il y a donc assurément une imprégnation johannique de l'alêtheia, mais il n'est pas nécessaire d'y voir une exception au principe général de l'adéquation: Jésus «est» certes la vérité dans le verset célèbre de Jn 14, 6 ( «Je suis le chemin et la vérité et la vie»), mais il la «dit» le plus souvent (Jn 8, 40; 8, 45-46; 16, 7).

L'issue de la discussion dépend pour une large part du statut à accorder aux nombreux «témoignages», marturia, dont il est question dans le texte de l'Évangile de Jean. Lorsque Jésus déclare que le témoignage qu' «un autre» lui rend est «vrai» (alêthês, Jn 5, 32), pourquoi la vérité dont il est ici question devrait-elle

9. Voir les deux grandes sources de Bultmann sur la question, Adolf Schlatter, Der Glaube im Neuen Testament. Eine Untersuchung zur neutestamentlichen Theologie (1885), Stuttgart, Calwer Verlag, $1982^{6}$ et Hans von Soden, «Was ist Wahrheit? Vom geschichtlichen Begriff der Wahrheit»(1927) [in] Id., Urchristentum und Geschichte. Gesammelte Aufsätze und Vorträge, t. 1, Grundsätzliches und Neutestamentliches, Tübingen, Mohr Siebeck, 1951, p. 1-24.

10. Ch. Landmesser, art. «Wahrheit/Wahrhaftigkeit», loc. cit., p. 341. 
différer de la vérité demandée à tout témoignage ? Celle-ci suppose la concordance du témoignage avec la réalité des faits, que le témoignage sert précisément à établir.

L'entretien avec la Samaritaine permet une comparaison. En validant la déclaration de la femme (Jn 4, 17-18: "“Je n'ai pas de mari" [...] "tu as dit vrai" », touto alêthes eirêkas), Jésus prend acte de sa conformité à la réalité d'une situation. Que la femme entende là la parole d'un «prophète» relève d'une titulature et d'une louange (tout comme «Seigneur» en Jn 4, 19), mais ne change rien à ce qu'alêthes désigne dans la bouche de Jésus: une correspondance entre des dires et des faits. À la suite, l'appel à «adorer Dieu en esprit et en vérité» (en pneumati kai alêtheia, Jn 4, 23-24), s'il ajoute le don de l'Esprit, semble toutefois dériver de ce premier constat d'adéquation.

La vérité du témoignage s'éprouve dans une règle en particulier, qui fixe dans la Torah le nombre et l'identité des témoins ${ }^{11}$. C'est cette règle qui est rappelée dans le verset cité de Jn 5, 32. «Un autre» rend témoignage à Jésus et si ce témoignage est dit vrai, c'est d'abord parce que c'est celui d' «un autre». L'anonymat ici - alors que le Père est nommé dans les versets qui suivent comme dans ceux qui précèdent - dirige l'attention vers la formalité de la règle, décrite par son contraire: «Si je me rendais témoignage à moi-même, mon témoignage ne serait pas vrai» (ean egô marturô peri emautou, hê marturia mou ouk estin alêthês, Jn 5, 31) ${ }^{12}$.

Telle est du moins la première rédaction de l'Évangile de Jean ${ }^{13}$. Les couches ultérieures du texte montrent Jésus violant au contraire

11. Dt 17, 6: «C'est sur les déclarations de deux ou trois témoins que celui qui doit mourir doit être mis à mort; il ne sera pas mis à mort sur les déclarations d'un seul témoin». Plus généralement Dt 19, 15: «Un témoin ne se présentera pas seul contre un homme qui aura commis un crime, un péché ou une faute, quels qu'ils soient; c'est sur les déclarations de deux ou de trois témoins qu'on pourra instruire l'affaire», et sur ce texte Hendrik Van Vliet, No Single Testimony. A Study on the Adoption of the Law of Deut. 19:15 par. into the New Testament, Utrecht, Kemink \& Zon, 1958. Cf. aussi Nb 35, 30. On peut aussi lire Jean-Pierre Charlier, «L'exégèse johannique d'un précepte légal: Jean viII 17», Revue Biblique, 67, 1960, p. 503-515.

12. Les sources rabbiniques de cette règle sont dans la Mishna, traités Roch Hachana 3, 1 et Ketouvot 2, 9: C. K. Barrett, The Gospel according to Saint John (1955), London, SPCK, $1962^{2}$, p. 338.

13. Sur les usages de marturia/marturein dans les différentes couches de rédaction du texte, voir la mise au point de W. Schmithals, Johannesevangelium und Johannesbriefe, op. cit., p. 304 et 346-348. 
la règle qui proscrit de témoigner pour soi-même : «Il est vrai que je me rends témoignage à moi-même, et pourtant mon témoignage est vrai [alêthês estin hê marturia mou], parce que je sais d'où je viens et où je vais » $(J n 8,14)$. Cette vérité alléguée du témoignage sur soi fonde la vérité des jugements que Jésus dit tenir parfois: «Moi, je ne juge personne; et s'il m'arrive de juger, mon jugement est vrai parce que je ne suis pas seul: il y a aussi Celui qui m'a envoyé» (Jn 8, 15-16). La transgression atteint son comble lorsque Jésus se met à compter: Jésus témoigne pour lui-même et son Père témoigne pour Jésus, Jésus réunit donc les deux témoins réglementaires de Dt 17-19. C'est une des provocations les plus stridentes de l'Évangile de Jean, un défi simultané à l'arithmétique et au droit:

Dans votre Loi il est d'ailleurs écrit que le témoignage de deux hommes est vrai [alêthês]. Je me rends témoignage à moi-même, et le Père qui m'a envoyé me rend témoignage lui aussi (Jn 8, 17-18).

Résumons. Les contradictions du texte, la violence de ses passages aux extrêmes, désignent dans le témoignage l'un des principaux lieux de problématisation de l'alêtheia dans Jean: le constat n'est certes pas nouveau ${ }^{14}$, mais il importe de le préciser. Le témoignage n'est pas le vecteur d'une conception alternative de la vérité qui donnerait congé à l'adéquation; les revirements du texte au fil de son assemblage révèlent plutôt que c'est le concept même de l'alêtheia qui est travaillé par autre chose, sans cesser de signifier l'adéquation.

De quoi s'agit-il? Les difficultés de traduction nous mettent sur la voie. Dans les versets cités, une version moderne comme la Traduction œcuménique de la Bible (TOB) hésite à traduire alêthês par «vrai», comme je viens de le faire à titre expérimental: elle propose tantôt « recevable » $(\mathrm{Jn} 5,31 ; 8,14 ; 8,17)$, tantôt « conforme à la vérité» $(\mathrm{Jn} 5,32 ; 8,16)^{15}$. À juste titre: en Jn 8,17 , le renvoi à la Torah ne signifie évidemment pas que tout témoignage produit

14. Vue d'ensemble dans Johannes Beutler, Martyria. Traditionsgeschichtliche Untersuchungen zum Zeugnisthema bei Johannes, Frankfurt a. M., Knecht (Frankfurter theologische Studien, 10), 1972. Cf. aussi dans la somme d'Ignace de La Potterie, La Vérité dans saint Jean, Rome, Biblical Institute Press, 1977 (19992), t. 1: Le Christ et la vérité. L'Esprit et la vérité, p. 79-116.

15. À titre de comparaison, c'est vrai en partie également pour une autre version moderne comme la English Standard Version, qui pour Jn 5, 31 propose: «If I alone bear witness about myself, my testimony is not deemed true» (je souligne). 
par deux hommes soit vrai, mais qu'il faut au moins deux témoins concordants pour instruire un cas et vérifier des faits, comme on l'a déjà vu dans le Tanakh ${ }^{16}$.

C'est donc le droit qui constitue l'épreuve première pour la vérité comme adéquation: la conformité du dire à l'être renvoie dans Jean à la conformité des faits au droit. Le témoignage johannique doit être pensé au regard d'une déposition. En cela, il est tout simplement biblique.

\section{Procès}

Nous disposons d'assez d'éléments pour ancrer la question de l'alêtheia dans le récit du procès:

Pilate rentra donc à la résidence. Il rappela Jésus et lui dit: «Es-tu le roi des Juifs?» Jésus lui répondit: «Dis-tu cela de toi-même ou d'autres te l'ont-ils dit de moi?»Pilate lui répondit: «Est-ce que je suis Juif, moi ? Ta propre nation, les grands prêtres t'ont livré à moi ! Qu'as-tu fait?» Jésus répondit: «Ma royauté n'est pas de ce monde. Si ma royauté était de ce monde, les miens auraient combattu pour que je ne sois pas livré aux Juifs. Mais ma royauté, maintenant, n'est pas d'ici». Pilate lui dit alors: «Tu es donc roi ?» Jésus lui répondit: «C'est toi qui dis que je suis roi. Je suis né et je suis venu dans le monde pour rendre témoignage à la vérité [hina marturêsô tê alêtheia]. Quiconque est de la vérité écoute ma voix». Pilate lui dit: "Qu'est-ce que la vérité?» [Ti estin alêtheia] (Jn 18, 33-38).

La première réponse, interrogative, de Jésus à Pilate («Dis-tu cela de toi-même ou d'autres te l'ont-ils dit de moi?») se souvient de l'accusation des Pharisiens en Jn 8, 13: «Tu te rends témoignage à toi-même! Ton témoignage n'est pas vrai !» (hê marturia sou ouk estin alêthês; la TOB traduit toujours : «recevable»). Dans le feu des questions échangées-retournées, ce sont les règles de la déposition qui restent débattues: nécessité de plusieurs témoins, impossibilité du témoignage pour soi. Le rappel régulier de ces règles révèle ici sa fonction dans l'ensemble du récit. En renvoyant à la prescription du Deutéronome sur le nombre de témoins à produire pour juger d'une accusation de crime capital, Jn 8 avait préfiguré la mise en croix. L'affront de Jésus aux Pharisiens avait alors appelé ce commentaire du narrateur, typique des annonces de la Passion dans Jean comme

16. Voir supra, n. 5-6. 
dans les Synoptiques: «Personne ne mit la main sur lui, parce que son heure n'était pas encore venue» (Jn 8, 20, cf. 12, 33; 18, 32, et pour le texte le plus ancien Mc 8, 31).

Cette fois pourtant, Jésus évite la transgression: sa réponse à Pilate («C'est toi qui dis que je suis roi», Jn 18, 37) fait écho au «Tu le dis» à Caïphe lors du procès devant le Sanhédrin, rapporté par les Synoptiques (Mt 26, 64; Lc 22, 70 ${ }^{17}$ ). Jésus répond donc sur la royauté des juifs comme il l'avait fait sur le Messie, et cette concordance des réponses relie les deux procès, religieux puis civil. Pour Soukkot il s'était exprimé sur les titulatures: «Qui parle de lui-même cherche sa propre gloire; seul celui qui cherche la gloire de celui qui l'a envoyé est vrai [alêthês; TOB : «véridique»] et il n'y a pas en lui d'injustice [adikia; TOB: «imposture»]» (Jn 7, 18). C'était la deuxième grande glose de l'Évangile de Jean sur le droit du témoignage (Jn 5, 31-32), après le commentaire immédiat de Jn 5, 45: «Comment pourriez-vous croire, vous qui tenez votre gloire les uns des autres et qui ne cherchez pas la gloire qui vient de Dieu seul?»

En somme, devant Pilate, Jésus témoigne mais ne dépose pas. D'un geste, sa marturia épouse et brise les contraintes du tribunal. La déposition de Jésus viendra ensuite, de la Croix, et Pilate la concédera aux disciples (Jn 19, 38-40).

\section{Tribunal}

Il est maintenant possible de formaliser. La déclaration: « Je suis né et je suis venu dans le monde pour rendre témoignage à la vérité [hina marturêsô tê alêtheia]. Quiconque est de la vérité écoute ma voix » $(\mathrm{Jn} 18,37)$ réunit deux composantes de la vérité-adéquation dans Jean: une scène et une relation.

1. La scène est celle du tribunal, civil ou religieux. Elle est dressée à l'énoncé des règles du témoignage en Jn 5. Quel que soit le sens de la si controversée question de Pilate, "Qu'est-ce que la vérité?» (Jn 18, 38), il n'y a aucun hasard à ce qu'elle soit posée au moment où Jésus est mené devant un juge. La question de la

17. Le récit dans Marc est en revanche catégorique: " "Es-tu le Messie, le Fils du Dieu béni ?" [...] "Je le suis"» (Mc 14, 61-62). Le débat sur l'auto-témoignage, relevé dans Jean, parcourt aussi les Synoptiques. 
vérité ne peut émerger que dans la recherche des chefs d'accusation qui devront guider l'établissement des faits. Elle est provoquée ou arrachée au juge par la marche contrariée de la procédure. Un juge ne pose la question de l'essence ( $t i$ esti), ne se fait donc philosophe que par irritation ou résignation, quand les réponses qu'il obtient le poussent dans ses retranchements de juge. «Qu'est-ce la vérité?» est une question de juge mis en échec.

Le juge est ici romain, mais le tribunal, qui est passé de Caïphe à Pilate, est romain et juif: pour les rédacteurs de l'Évangile de Jean, le droit est rabbinique comme il est romain. C'est pour cette raison que l'alêtheia johannique reste si profondément biblique. Elle est héritière de la vérité salomonienne des Proverbes, qui «fait éclater» la justice quand le «faux témoin» ne crée que fausseté $(\operatorname{Pr} 12,17)$; héritière de la vérité du juste dans les Psaumes, qui «dit la vérité en son cœur», c'est-à-dire comme il la pense (Ps 15 [14], 2); héritière de la vérité de l'Exode, quand Jéthro, beau-père de Moïse, l'enjoint à «discern[er] [...] des hommes de valeur, vrais [TOB: «dignes de confiance»], incorruptibles» et à les «établi[r] comme chefs» sur Israël: «ils jugeront le peuple en permanence» (Ex 18, 21-22). L'alêtheia dans Jean sort du droit par le droit.

Dans la diversité des occurrences (sincérité du témoin, fiabilité des juges), l'épreuve de vérité est unique : c'est celle de la collation des correspondances entre faits, paroles et pensées, sur le modèle de l' "enquête» dont il est question dans le Deutéronome ${ }^{18}$. Cette épreuve est toujours située: le tribunal est le lieu biblique de l'adaequatio rei et intellectus, jusqu'à Jean inclus.

2. La relation est celle déjà vue du témoignage, à proprement parler relation des faits en vue de leur établissement. La formule de Jn 18, 37, «rendre témoignage à la vérité», condense les débats rapportés en Jn 5 et 8. Elle est désormais synthétique car complète: le Fils rend témoignage de ce que le Père lui a rendu témoignage. Après l'affirmation de sa royauté venue d'ailleurs («ma royauté n'est pas de ce monde», Jn 18,36), Jésus peut valider la transgression des règles du témoignage opérée en Jn 8, 18 ( «Je

18. Dt 13, 16, répression de l'idolâtrie: «[...] tu feras des recherches, tu t'informeras, tu mèneras une enquête approfondie; et une fois vraiment établi le fait que cette abomination a été commise au milieu de toi, tu frapperas du tranchant de l'épée tous les habitants de cette ville». Sur ce texte, cf. Jürgen van Oorschot, art. «Wahrheit/Wahrhaftigkeit», Theologische Realenzyklopädie, loc. cit., p. 338. 
me rends témoignage à moi-même, et le Père qui m'a envoyé me rend témoignage lui aussi»). La relation qu'il décrit est tout à la fois parfaitement réciproque et parfaitement hiérarchique, livrant une forme irreprésentable aux Pharisiens comme au fonctionnaire romain: celle de toute la «dogmatique chrétienne $»^{19}$.

\section{ReLATions}

Vouloir ressaisir le mystère de ce dogme à partir d'une pratique ou d'un acte de parole aussi ordinaire que celui du témoignage de tribunal apparaîtra sans doute comme une gageure. Assurément, la relation de témoignage dans Jean se déploie à l'intérieur d'un vaste réseau de notions, qui inclut par exemple la gloire (doxa, cf. Jn 7 , $18 ; 8,54)$. La continuité est ainsi garantie avec la spéculation du Prologue, où déjà Jean le Baptiste «rend témoignage à la Lumière » $(\mathrm{Jn} 1,7)$ et à la «gloire» du Verbe fait chair $(\mathrm{Jn} 1,14)$. Le réseau ne tient toutefois que par le lien premier qui rattache le témoignage à l'alêtheia, ou plutôt qui fait de la vérité l'issue recherchée d'un témoignage. Le Prologue de Jean trouve en Jn 8 son explicitation, sa simplification peut-être, à usage polémique contre la Synagogue, mais surtout sa vérification, l'établissement de sa vérité à travers la procédure juridique du témoignage.

De la même façon, les relations décrites dans l'Évangile de Jean se diversifient à partir de l'alêtheia. Celle-ci recouvre en particulier un lien d'appartenance ou d'inclusion qui fait de la vérité un lieu où l'on se tient aussi bien qu'une propriété que l'on contient (Jn 8, 44: le diable «ne s'est pas tenu dans la vérité parce qu'il n'y a pas en lui de vérité» et à l'inverse Jn 10,38: «[...] afin que vous connaissiez et que vous sachiez bien que le Père est en moi comme je suis dans le Père » $)^{20}$. C'est encore de cette appartenance qu'il est question devant Pilate: «Quiconque est de la vérité [ek tês alêtheias] écoute ma voix»

19. On se reportera à la remarque de Jean-Luc Marion à partir de l'énoncé de confession de foi de Rm 10, 9, «Jésus est Seigneur»: «la dogmatique chrétienne assure cette qualification en reconnaissant [à Jésus] la divinité, ou mieux en reconnaissant que le Père la lui a sans cesse reconnue» (Jean-Luc Marion, Dieu sans l'être [1982], Paris, PUF [Quadrige], 1991², p. 265). En remplaçant la reconnaissance dont parle Marion par le témoignage, on obtient très exactement le schéma qui achève de se dessiner dans les paroles de Jésus devant Pilate.

20. Cf. I. de La Potterie, La Vérité dans saint Jean, op. cit., t. 2, p. 593-635. 
(Jn 18, 37). Que cette formule succède de nouveau à la mention d'un marturein (hina marturêsô tê alêtheia) en dit suffisamment long sur le caractère dérivé, dans Jean, de la relation d'inclusion par rapport à la relation de témoignage. Les dernières occurrences d'alêtheia dans l'Évangile de Jean concentrent les difficultés autour du «disciple que Jésus aimait» $(\mathrm{Jn} 21,20)$ et de son identification, mais elles sont parfaitement claires quant à la signification ordinaire de la vérité dans le témoignage. Il s'agit simplement de convaincre, comme à la descente de croix: "Celui qui a vu a rendu témoignage, et son témoignage est véridique [alêthinê; TOB : «conforme à la vérité»], et d'ailleurs celui-là sait qu'il dit ce qui est vrai [oiden hoti alêthê legei] afin que vous aussi vous croyiez» (Jn 19, 35, cf. 21, 24).

Résumons: dans Jean comme dans le reste des écrits bibliques, le droit est la ratio cognoscendi de la nature du vrai. Jamais le texte ne rompt complètement avec le sens ordinaire de ce qu'est un témoignage concordant avec des faits, y compris lorsqu'il s'agit d'affirmer l'incroyable, à savoir que la vérité du témoignage de Jésus provient du témoignage que lui rend son Père. L'extrême violence auxquelles sont soumises les règles du témoignage, de l'intérieur du droit rabbinique (Jn 8), le détournement, de l'intérieur aussi, de la procédure d'interrogatoire tentée par Pilate avant la mise en croix (Jn 18), tout le montre: le droit est à la fois point de repère et point de rupture. Il faut envisager les conséquences de cette double position.

\section{Comme IL DIT}

Dans un livre récent sur Pilate et Jésus, Giorgio Agamben s'est livré à un rappel salutaire:

L'affirmation de Jésus sur le témoignage de la vérité [= Jn 18, 37, «Je suis né et je suis venu dans le monde pour rendre témoignage à la vérité»] a été souvent tenue pour énigmatique ou du moins telle que Pilate ne pouvait la comprendre. La phrase, restituée dans son contexte, n'a pourtant rien d'énigmatique. Jésus se trouve dans un procès devant un juge qui l'interroge, et le fait de témoigner de la vérité est ce qu'on attend de tout accusé et de tout témoin. ${ }^{21}$

21. Giorgio Agamben, Pilato e Gesù (2013), Roma, Nottetempo, 2014², p. 59, tr. fr. J. Gayraud, Pilate et Jésus, Paris, Payot \& Rivages, 2014, p. 73. L'interprétation suppose une réduction radicale de la doctrine des deux natures: 
Il se pourrait donc que les choses soient encore plus simples qu'on ne les a présentées ici et que "témoigner de la vérité» ne signifie rien d'autre que «dire ce qui est» dans le cadre réglementé d'un procès. En l'occurrence cependant, le procès a échoué. Agamben, passé un rappel presque obligé des interprétations de la question de Pilate ${ }^{22}$, ne se préoccupe guère de la signification de cet échec pour la compréhension de l'alêtheia.

C'est pourtant bien Agamben qui insiste sur ce point: en toute rigueur juridique, il n'y a pas eu de procès de Jésus, car il n'y a pas eu de jugement; Jésus a seulement été «livré» aux juifs, sans autre forme de procès ${ }^{23}$. L'auteur en tire plusieurs conclusions sur la disjonction et l'indiscernabilité de la justice et du salut, de l'éternel et du temporel, du jugement et de la vie ${ }^{24}$. Essentiellement, c'est le vieux concept d'une «économie» ou histoire du salut qui est remis en service ${ }^{25}$.

Agamben relie l'absence de jugement de Jésus (ou son «simulacre de procès $»^{26}$ ) à l'affirmation, en Matthieu, que Jésus ne juge pas (Mt 7, 1: «Ne jugez pas, pour ne pas être jugés») ${ }^{27}$. C'est pour le coup faire peu de cas de la singularité johannique. Il est vrai que dans Jean «Dieu n'a pas envoyé son Fils dans le monde pour juger le monde, mais pour que le monde soit sauvé par lui» (Jn 3, 17), mais Jésus, on l'a vu, dit expressément qu'il «juge» parfois. Le régime de ses jugements est rigoureusement aligné sur celui de ses témoignages (Jn 8, 16, «et s'il m'arrive de juger, mon jugement est conforme à la vérité parce que je ne suis pas seul», cf. 8, 14) et, peut-on penser, sur l'alêtheia qui y est énoncée. Les rapidités

«Quand Jésus dit à Pilate qu'il est venu au monde pour témoigner de la vérité, il n'entend certes pas dire qu'ayant deux natures et deux volontés, avec l'une d'elles - l'humaine - il témoigne de l'autre - la divine (et vice-versa). Sa tâche serait bien trop facile. [...] Lorsque Jésus est homme, il est homme et c'est tout, exactement comme Pilate».

22. Ibid., p. 30-31, tr. fr. p. 36-38, à partir de Nietzsche et Spengler.

23. Ibid., p. 67-68, tr. fr. p. 83-84 et p. 42-43, tr. fr. p. 52-54 sur la «livraison» (paradosis) de Jésus.

24. Ibid., p. 63, 65, 74, tr. fr. p. $78,81,92$.

25. Voir par exemple ibid., p. 45, 50-51, tr. fr. p. 55, 62-64 et bien sûr G. Agamben, Il regno e la gloria. Per una genealogia teologica dell'economia e del governo (Homo Sacer, II, 2), Vincenza, Neri Pozza, 2007, tr. fr. J. Gayraud et M. Rueff, Le Règne et la gloire, Paris, Le Seuil, 2008.

26. G. Agamben, Pilato e Gesù, op. cit., p. 44, tr. fr. p. 54.

27. Ibid., p. 66-67, tr. fr. 53-54. Agamben semble tenir pour acquis que Jésus s'applique cet interdit. 
d'Agamben sur ce point me semblent ainsi renvoyer à une question plus fondamentale sur le devenir du droit dans le christianisme et sur son lien avec la revendication de vérité.

Je m'explique. À partir de Jn 5 au plus tard, Jésus pense et débat dans les formes du procès pour défaire la formalité du droit. Il y a là davantage qu'une «objection» au droit ou un «règlement de comptes» avec la loi, comme le dit Agamben en considérant la critique paulinienne dans l'épître aux Romains ${ }^{28}$; davantage aussi, évidemment, qu'une critique du prétendu juridisme d'Israël. Quelques décennies après la mort de Paul, les auteurs de l’Évangile de Jean ont épousé au plus près les contours officiels du témoignage, du procès et du droit. Ils ne l'ont pas fait seulement pour «désactiver» la $\mathrm{Loi}^{29}$, mais pour suggérer que la vérité de ce qu'ils avaient à dire pouvait se comprendre, bien qu'elle n'eût rien de commun avec celle que les hommes revendiquent.

\section{LE TOURNANT ECCLÉSIOLOGIQUE DE LA PHILOSOPHIE CONTEMPORAINE}

En somme, au prix d'hésitations quant au statut du témoignage, l'Évangile de Jean est parvenu à la plus parfaite cö̈ncidence entre le droit et son contraire. Il serait souhaitable de tirer de cela quelques conclusions. La question de l'anti-juridisme chrétien est à remettre à l'agenda de la philosophie politique et juridique, car il est devenu urgent de se convaincre qu'en dépit d'Agamben ou de Legendre ni le droit canon, ni l'Église, ni même l' «institution» ou l'officium ne sont le code unique de l' «Occident», s'il existe. Une critique de l'étrange tournant, non plus seulement théologique mais ecclésiologique, de la philosophie contemporaine s'impose aujourd'hui avec une certaine insistance. Nous savons cependant qu'elle ne suffira pas. La tâche est d'identifier ce contraire du droit que le christianisme a voulu loger au cœur du droit, du témoignage et de ses actes de parole, et de savoir ce que nous voudrons en faire.

28. Ibid., p. 70-71, 76, p. 88-89, 94.

29. Il faut toujours renvoyer à l'analyse très neuve d'Agamben sur la désactivation de la Loi chez Paul, en Rm 3 et 7, et sur le sens quasi-dialectique (Aufhebung!) de kathargein: cf. G. Agamben, Il tempo che resta. Un commento alla Lettera ai Romani, Torino, Bollati Boringhieri, 2000, p. 91-96, tr. fr. J. Revel, Le Temps qui reste. Un commentaire de l'Épître aux Romains, Paris, Payot \& Rivages, p. 152-161. 
Provisoirement, je proposerai ceci : homologia - la confession de la foi comme relais de la marturia, du témoignage, jusqu' au moment où celui-ci aura rompu ses liens avec le procès. Telle est l'attente chrétienne, celle d'un temps où il sera possible de tenir un discours aussi libre que celui que Jésus, pour lui-même, oppose à Caïphe: «J'ai parlé ouvertement au monde» (egô parrêsia lelalêka tô kosmô, Jn 18, 20). Ce libre «enseignement» (egô pantote edidaxa) trouve son pendant temporaire dans la «confession» demandée à chacun par la première épître de Jean ${ }^{30}$. L'homologia est le régime de vérité qui laisse entrevoir l'adieu aux procédures du droit; elle garde sa place au cœur des Églises et de leurs liturgies: credo. En elle les traits les plus singuliers de l'alêtheia johannique se réunissent à la parole des hommes: 1'esprit (1 Jn 4, 2: «À ceci vous reconnaissez l'Esprit de Dieu: tout esprit qui confesse [homologei] Jésus Christ venu dans la chair est de Dieu »), l'inclusion (1 Jn 4, 15 : «Quiconque confesse que Jésus est le Fils de Dieu, Dieu demeure en lui et lui en nous»).

Mais cette langue-là est-elle vraiment pour nous? Une «religion», même, peut-elle en vouloir? Jean et toute la Bible enseignent que le droit est le régime ordinaire du vrai, au regard duquel se constate toute exception au «concept courant de la vérité». Les écrits bibliques laissent aussi deviner autre chose. Que seraient une vérité et une langue sans droit? Souhaitons-nous vraiment le savoir? Il est heureux, peut-être, que la chose ne soit pas censée dépendre de nous.

Philippe.Buettgen@univ-paris1.fr

30. Sur l'homologia dans le corpus johannique, voir J. Beutler, «Faith and Confession: The Purpose of John» (2002), [in] Id., Neue Studien zu den johanneischen Schriften, Göttingen-Bonn, V\&R Unipress-Bonn University Press (Bonner biblische Beiträge, 167), 2012, p. 101-114. 